\title{
Outbreak of wound botulism in injecting drug users
}

\author{
M. SCHROETER ${ }^{1}$, K. ALPERS ${ }^{2 *}$, U. VAN TREECK ${ }^{1}$, C. FRANK ${ }^{2}$, \\ N. ROSENKOETTER ${ }^{1}$ AND R. SCHAUMANN ${ }^{3}$ \\ ${ }^{1}$ Institute for Public Health, North Rhine-Westphalia, Bielefeld and Münster, Germany \\ ${ }^{2}$ Department for Infectious Disease Epidemiology, Robert Koch-Institute, Berlin, Germany \\ ${ }^{3}$ Institute for Medical Microbiology and Epidemiology of Infectious Diseases, National Reference Laboratory for \\ Anaerobes, University of Leipzig, Leipzig, Germany
}

(Accepted 6 March 2009; first published online 7 April 2009)

\section{SUMMARY}

Between October and December 2005, 16 cases of wound botulism were notified to the health authorities of North Rhine-Westphalia, Germany. All patients were injecting drug users (IDU) and the epidemiological investigations suggested contaminated injection drugs as the most probable source of infection. Clostridium botulinum was cultivated from clinical samples of six patients and molecular typing revealed that the different isolates were clonally identical. Two samples of heroin, one of them provided by a patient, were examined but $C$. botulinum could not be isolated. This outbreak demonstrates that IDU are at risk for acquiring wound botulism by injecting contaminated drugs. A greater awareness of this disease is needed by physicians and a close cooperation between public health authorities, street workers, operators of sheltered injecting facilities, and medical centres focusing on IDU is essential to prevent and manage outbreaks in IDU in a timely manner.

Key words: Clostridium botulinum, drug abuse, heroin, outbreak investigation, parenteral molecular typing, wound botulism.

\section{INTRODUCTION}

Botulism is a rare disease, caused by neurotoxin release from the anaerobic spore-forming bacteria Clostridium botulinum. Three main, naturally occurring epidemiological types of botulism can be distinguished: foodborne, intestinal colonization (infant form) and wound botulism. In Germany, botulism is a notifiable disease. Between 2001 and 2004, 33 cases of botulism were notified to the German health authorities (6-11 per year) of which 29 were considered to

\footnotetext{
* Author for correspondence: Dr K. Alpers, Abteilung für Infektionsepidemiologie, Robert Koch-Institut, Seestraße 10, D-13353 Berlin.

(Email: AlpersK@rki.de)
}

be foodborne in adults, three were infant botulism, and one wound botulism [1].

In the days leading up to 3 November 2005, nine cases of wound botulism in injecting drug users (IDU) were notified in North Rhine-Westphalia (NRW). An investigation was initiated to identify the extent and the source of the outbreak in order to prevent the occurrence of more infections. In this paper we describe the epidemiological and laboratory investigations conducted.

\section{METHODS}

\section{Epidemiological investigations}

Case-patients were defined as current IDU, resident in Germany, presenting with acute onset of flaccid 
paralysis or cranial nerve palsies, from 1 October 2005 onwards. Once their clinical condition allowed, patients who gave informed consent were interviewed by health authority personnel or trained social workers with a detailed standardized questionnaire containing questions on clinical symptoms, consumption of food known to bear a risk for transmission of botulism, drug-consumption habits during the 4 weeks prior to hospital admission, and whether the patients knew about other cases. Patients were also asked whether they bought their heroin in Germany or in the adjacent Netherlands and whether they knew from which country or region the heroin originated.

On 14 November 2005 a message was circulated to all public health departments and emergency physicians in NRW via email networks maintained by the Institute for Public Health, NRW and the local public health departments of NRW. Via the German national Epidemiological Bulletin physicians and public health authorities in other German federal states were informed about the occurrence of wound botulism in IDU in NRW [2, 3]. Through organizations offering outreach, sheltered injecting facilities, and via street workers attempts were made to inform the drug-using communities in Germany about the clinical symptoms of botulism as well as possible routes for acquiring the disease. IDU were asked to seek medical advice if symptoms (flaccid paralysis, difficulties in swallowing, blurred vision) were present. Physicians and laboratories were reminded to immediately notify any cases of botulism.

Due to possible cross-border spread of the outbreak, information was also distributed through the European Early Warning and Response System as well as Eurosurveillance [4].

\section{Laboratory investigation}

Wound swabs obtained from six patients were sent to the National Reference Laboratory for Anaerobes (NRL-A) for further identification.

As illicit injection drugs were the most strongly suspected source of infection, patients were asked for samples of heroin they were using in order to investigate it for contamination with Clostridium spores.

\section{Culture and identification of clinical samples}

At NRL-A wound swabs were analysed employing Columbia agar (Oxoid Ltd, UK) supplemented with $5 \%$ sheep blood (Oxoid GmbH, Germany), vitamin
K1 (Sigma Chemical Co., USA), and haemin (Serva Feinbiochemica, Germany), as well as standard appropriate supplemented broth media under anaerobic conditions to cultivate anaerobes. Standard appropriate solid und broth media were used for aerobic cultures to control possible aerobe growth. The anaerobic cultures were incubated in an anaerobic chamber (Heraeus, Germany) containing 80\% $\mathrm{N}_{2}$, $15 \% \mathrm{CO}_{2}$ and $5 \% \mathrm{H}_{2}$ at $37{ }^{\circ} \mathrm{C}$. Obligate anaerobic strains were identified by the Rapid ID 32A system (bioMérieux, France) and by using pre-reduced anaerobic systems (PRAS; Anaerobe Systems, USA).

\section{Cultivation of heroin samples}

Two heroin samples were evaluated for microbiological culture. The samples were divided into several aliquots and thereafter added to brain heart infusion broth (Oxoid Ltd) supplemented with vitamin K1 and haemin. Samples were either untreated or heat-shocked and incubated at $36{ }^{\circ} \mathrm{C}$ for 3 weeks under aerobic and anaerobic conditions. Subcultures were performed on days 7, 14, and 21 or after visible growth on appropriate agar plates as described above and incubated under aerobic and anaerobic conditions.

\section{Characterization and comparison of isolates}

$16 \mathrm{~S}$ rDNA amplification was performed with the primers $609 \mathrm{~V}$ and 699R as described by Ackermann et al. [5] resulting in a PCR product of $\sim 300 \mathrm{bp}$. Amplification sequences were compared using the NCBI BLAST database (http://www.ncbi.nlm.nih. gov/blast/Blast.cgi).

For PCR, DNA was prepared using the Qiagen tissue kit (Qiagen GmbH, Germany) according to the manufacture's instructions. PCR assays were separately performed for $C$. botulinum type A, B, E, and F neurotoxin genes with the specific primers AS-11 and AS-22 (toxin type A; sequences described by Takeshi et al. [6]) to produce a PCR product of $\sim 283 \mathrm{bp}$ and the specific primers CBMLB1, CBMLB2, CBMLE1, CBMLE2, CBMLF1, and CBMLF2 (toxin types B, E, and F; sequences described by Lindström et al. [7]) to produce a PCR product of $\sim 205 \mathrm{bp}$ (toxin type B), 389 bp (toxin type E), and 543 bp (toxin type F). After 2 min of denaturation at $95^{\circ} \mathrm{C}, 35$ cycles of the PCR mix with a final volume of $50 \mu \mathrm{l}$ were run as follows: $30 \mathrm{~s}$ at $94{ }^{\circ} \mathrm{C}, 30 \mathrm{~s}$ at $55^{\circ} \mathrm{C}$ for toxin types B, E, and $\mathrm{F}$, and $30 \mathrm{~s}$ at $53{ }^{\circ} \mathrm{C}$ for toxin type $\mathrm{A}$, respectively, $1 \mathrm{~min}$ 
at $72{ }^{\circ} \mathrm{C}$ and a final extension cycle of 3 min at $72{ }^{\circ} \mathrm{C}$. C. botulinum reference strains (REB 1750, toxin type A; REB 89, toxin type B; REB 1072, toxin type E; and REB 1718, toxin type F) were used as control for each toxin type.

For the antibiogram the susceptibility of the isolates to penicillin, clindamycin, piperacillin/tazobactam, imipenem, and metronidazole was determined by Etest (AB Biodisk, Sweden), performed according to the manufacturer's instructions [8].

Serum samples from eight patients were tested in bioassays on mice. Briefly, $0.5 \mathrm{ml}$ serum of a patient with suspected wound botulism was administered intraperitoneally to two mice. In the control group of two other mice, each was initially administered intraperitoneally $0.5 \mathrm{ml}$ antitoxin (Botulism Antitoxin Behring; $1 \mathrm{ml}$ contains: 750 IU C. botulinum type A antitoxin, $500 \mathrm{IU} C$. botulinum type B antitoxin, 50 IU C. botulinum type E antitoxin; Chiron Behring GmbH \& Co., Germany) followed by $0.5 \mathrm{ml}$ serum of the patient $30 \mathrm{~min}$ later. The mice were observed until day 7 post-injection for survival and clinical signs of botulism.

PFGE was performed by contour-clamped homogenous electric field electrophoresis (CHEF-DR III apparatus; Bio-Rad, Germany) using the GenePath Group 1 Reagent kit (Bio-Rad Laboratories, USA) according to the protocol described previously for the GenePath Group 3 Reagent kit and SpeI [9]. Restriction digestion was performed with SmaI (BioRad Laboratories). For PFGE interpretation the criteria described by Tenover et al. were applied [10].

Different control strains were used: a $C$. botulinum isolate carrying the neurotoxin A gene and additionally a $C$. botulinum strain carrying the neurotoxin B gene (reference strain REB 89). A C. sporogenes strain served as control for closely related other Clostridium species. The $S$. aureus strain (NCTC 8325) was used as a culture control with the GenePath Group 1 kit.

\section{RESULTS}

\section{Epidemiological investigation}

Between 13 October and 5 December 16 cases occurred. After information on the outbreak had been distributed at local, national, and European level, six cases which initially had not been notified came to the attention of the German health authorities. One patient self-reported following hospitalization with classical symptoms retrospectively classified as botulism, but misdiagnosed as a drug overdose. Cases from outside Germany were not reported.

Of the 16 case-patients 12 were male and four female. Median age was 33 years (range $20-50$ years). Cases resided in eight different districts of NRW which are in close proximity to each other (Fig. 1). Eleven of the cases lived in cities (Cologne, Bonn, Aachen, Krefeld), the remainder in adjacent rural districts. The most common symptoms reported were difficulties in breathing and swallowing [14 cases (88\%) and 9 cases (56\%), respectively], and blurred vision [11 cases $(69 \%)]$. Cranial nerve palsy, ptosis, and palsy of peripheral nerves were observed in nine $(56 \%)$, six $(38 \%)$, and four $(25 \%)$ cases, respectively. All patients were hospitalized (median duration 26 days) and recovered fully. Assisted ventilation was required by five $(31 \%)$, and administration of antitoxin also by five patients. All patients were IDU, injecting heroin on a regular basis. Twelve reported injecting drugs subcutaneously and/or intramuscularly. The other four denied this route of injection, but nevertheless clinical examination of their skin revealed changes (circular scars, swellings, and redness) which could be due to subcutaneous injection ('skin popping').

Nine cases (six males) were interviewed with the study questionnaire while the other seven refused to participate. One of the nine questionnaires was excluded from analysis because it largely contained missing or indeterminate answers. Two respondents answered that they knew about further cases; however, they were unable to provide names or addresses for these persons. No meals or gatherings were attended by two or more patients. Food items known to bear a risk for transmission of botulism, including home-bottled or vacuum-packed foods, had not been consumed recently. Six patients had been using drugs for a median length of 12 years (range 2-35 years). Two patients did not specify how long they had used drugs previously. However, one of them had used drugs for 'several years' and both indicated that they had injected at least daily the month before the infection.

The heroin consumed by the patients before onset of symptoms came from different sources and was reported to be of different colours (ranging from pale to dark brown) and consistencies - two patients described a black-brown resinous consistency. Five respondents had used dark-coloured heroin within the 4 weeks before symptom onset, whereas the other three said, that they used 'normal' heroin without 


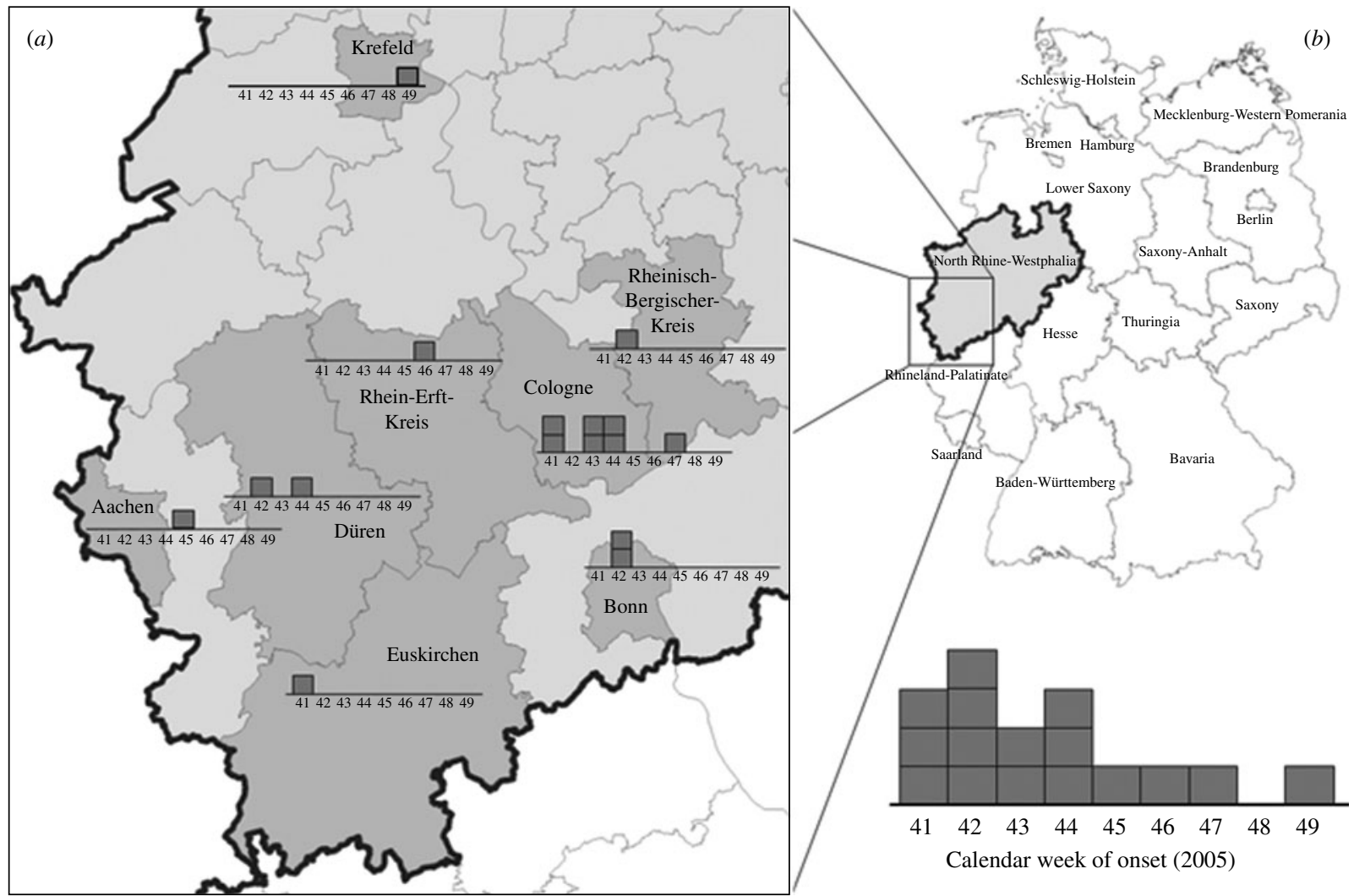

Fig. 1. (a) Cases of botulism in injecting drug users (IDUs) by residence and week of onset; (b) Cases of botulism in IDUs by week of onset $(n=16)$, Germany, 2005.

further specification. A traceback was not possible. Two patients reported buying heroin in The Netherlands themselves, and two others had bought their heroin from dealers who had bought it in The Netherlands. The other four had recently changed dealers and did not know the origin of the heroin sold to them. None of the patients had recently shared needles or syringes. None had changed their typical sources of water, acidifying agents (ascorbic acid and lemon juice) and other ingredients for the preparation of the drugs.

\section{Laboratory investigation}

In six patients $C$. botulinum were isolated from wound swabs. In one case bacteria were isolated from different wounds and appeared macroscopically as two morphologically distinct isolates. However, by PFGE analysis the strains were indistinguishable. In three wound swabs, aside from C. botulinum, cultures of normal skin flora were obtained, i.e. viridans streptococci and coagulase-negative Staphylococcus spp. From one specimen $S$. aureus was cultivated. No other Clostridium spp. were detected. The MIC ranges $(\mu \mathrm{g} / \mathrm{ml})$ of the $C$. botulinum strains were: $0 \cdot 06-0 \cdot 25$ penicillin, $4 \cdot 0-8 \cdot 0$ clindamycin, $0 \cdot 5-4 \cdot 0$ piperacillin/ tazobactam, $0 \cdot 06-0 \cdot 125$ imipenem, and $0 \cdot 25-4 \cdot 0$ metronidazole.

The neurotoxin B gene was detected by PCR in all of these strains whereas PCR was negative for neurotoxin $\mathrm{A}, \mathrm{E}$, and $\mathrm{F}$ genes. All seven strains investigated by PFGE exhibited an identical clonal profile (Fig. 2). In contrast, the two C. botulinum control strains showed distinct patterns.

Serum samples were obtained for mouse bioassays from three patients with a positive culture of C. botulinum and from additional five patients with either a negative culture result or without available material for culture. None of the bioassays were unequivocally positive.

Only one patient was willing to submit a heroin sample from his personal stock for testing. A second sample was obtained from the police. This sample had been confiscated in Cologne and although it is unclear whether this sample bears any relation to the outbreak, both samples were examined but were repeatedly culture negative for $C$. botulinum. Only Propionibacterium spp., Enterococcus spp., and 


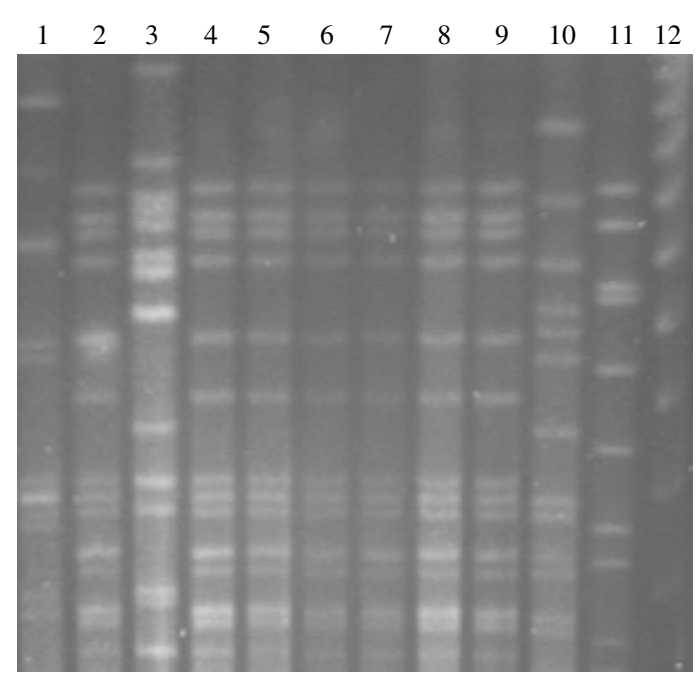

Fig. 2. PFGE pattern of seven C. botulinum strains isolated from six patients during the outbreak and control strains. Lane 12, $\lambda$-ladder (50-1000 kb); lane 11, S. aureus control strain of the PFGE kit; lanes 1, 10, C. botulinum control strains (lane 1, neurotoxin A gene-carrying strain; lane 10, neurotoxin B gene-carrying strain); lane 3, C. sporogenes; lanes 2, 4-9, C. botulinum strains from the six patients.

coagulase-negative Staphylococcus spp. could be cultivated.

\section{DISCUSSION}

We describe the first outbreak of wound botulism in Germany. Although the definitive source could not be determined, the finding of clonality in PFGE analysis of seven C. botulinum isolates derived from six patients strongly suggests a common source of the outbreak. Contaminated heroin seems to be most probable vehicle since other links between the cases (such as shared needles or dilution materials) could not be identified.

The negative culture result of the single heroin sample obtained from a case does not exclude heroin as a probable vehicle because street heroin has been described as having an antibacterial effect and the number of positive cultures depends on the preanalytical preparation methods in the laboratory and the culture conditions of the heroin samples [11, 12]. In addition, the sample may not be from the same batch as the heroin at the source of the outbreak.

The investigation of this outbreak was hampered by legal restrictions concerning the usage of confiscated heroin samples for purposes other than criminal prosecution. A critical review of this issue by the district government responsible came to the conclusion that even heroin confiscated in the context of a criminal prosecution cannot be readily provided for other purposes such as microbiological investigations.

The negative mouse bioassay results of the serum samples of eight patients might be explained by late collection of serum samples, although the samples were collected prior to treatment with antitoxin. This is known to be an important factor, because the toxin cannot be detected in serum once it permanently becomes bound to its cell receptors [13]. Furthermore, toxin type A is more aggressive than toxin type B [14]. Thus, the free and unbound amount of toxin present in the serum was probably below the level of detection. Toxin testing was positive only for $37 \%$ of sera collected from 309 persons with clinically diagnosed foodborne botulism reported to CDC from 1975 to 1988 [15]. Critchley et al. reported on 27 patients in an outbreak of foodborne botulism in North West England and Wales in 1989. All serological tests of botulism in the affected patients were negative [16]. On the other hand, a study in the UK showed that $33 / 88$ cases of wound botulism in heroin injectors $(37.5 \%)$ reported in the UK and Ireland between 2000 and 2004 were laboratory confirmed by the detection of botulinum neurotoxin in serum [17]. Furthermore, Akbulut et al. reported the detection of botulinum neurotoxin by bioassay in the sera of $9 / 16$ $(56 \cdot 3 \%)$ patients with suspected botulism who were IDU [18].

In the late 1990s wound botulism had become an increasing problem in California and subsequently became apparent elsewhere in the USA $[19,20]$. In Europe, an increase of soft-tissue infections with spore-forming bacteria (Clostridium and Bacillus spp.) was reported from the UK starting in $2000[13,21]$. In those reports the disease was linked to IDU injecting drugs by muscle or skin 'popping' [21]. In the USA, the intramuscular or subcutaneous usage of 'black tar heroin' (BTH), a dark, gummy and frequently impure heroin product primarily from Mexico and countries south of the USA, was described to be particularly associated with wound botulism [19, 20, 22, 23]. Due to its tarry consistency, this form of heroin is injected mainly subcutaneously or intramuscularly which provides excellent environmental conditions for the spores to germinate to bacteria. BTH does not seem to be the reason for the increase of disease in the UK since the heroin distributed in Europe primarily originates from Asia $[24,25]$. However, different forms of heroin may be injected subcutaneously or intramuscularly, especially 
by IDU experiencing difficulties in injecting intravenously. Acidifying agents required to dissolve the heroin can cause tissue damage and thus create favourable conditions for the growth of anaerobic bacteria.

All of the IDU in this outbreak either reported subcutaneous or intramuscular injection of heroin or showed clinical signs of this practice. Five survey respondents mentioned using heroin which appeared to them to be of an unusually dark colour. However, this information could not be verified and therefore does not allow us to conclude whether this heroin was actually BTH or not.

Parenteral drug use is well known for carrying the risk of transmission of several infectious diseases [26-29]. Wound botulism is of special importance for two reasons: first, the clinical course is often severe and life-threatening, and second, the rarity of the disease renders diagnosis difficult. Furthermore, in IDU the disease is often misdiagnosed as drug-related complications, a problem which also occurred in this outbreak. The correct and timely diagnosis of botulism in IDU is often complicated by multiple health problems due to malnutrition, poor hygiene and underlying co-infections. In addition to the harmful effect on the health of those concerned, the need for intensive care and treatment costs also have repercussions on the health-care system. A greater awareness of this disease is needed by physicians, as early diagnosis and prompt therapy are vital for the reduction of both the risk for death and duration of hospitalization [30]. If IDU choose to inject drugs into muscle or skin they should be made aware of the dangers of wound botulism in order to help detect the disease early on.

Illicit drug use remains an important issue for public health authorities in the future [31, 32]. Close cooperation between public health authorities, street workers, operators of sheltered injecting facilities, and medical centres focusing on IDU is essential to establish a network able to spread information about newly emerging health threats swiftly and to establish hazard reduction measures quickly in this high-risk group for infectious diseases.

\section{ACKNOWLEDGEMENTS}

We acknowledge the helpful contributions and the excellent cooperation of our numerous colleagues in the local health departments, the regional laboratories and all notifying physicians during the outbreak.

\section{DECLARATION OF INTEREST}

None.

\section{REFERENCES}

1. Robert Koch-Institut. Annual report on reportable communicable diseases for 2004 [in German]. Berlin: Robert Koch-Institut, 2004, pp. 50-51.

2. Treeck van U. Regarding an outbreak of wound botulism in North-Rhine-Westphalia [in German]. Epidemiologisches Bulletin 2005; 45 : 420.

3. Treeck van U, Alpers K. Update to the outbreak of wound botulism among injecting drug users in NorthRhine-Westphalia [in German]. Epidemiologisches Bulletin $2005 ; 48: 453$.

4. Alpers K, van Treeck U, Frank C. Outbreak of wound botulism in injecting drug users in Germany, October-December 2005. Eurosurveillance Weekly Release 2005; 12.

5. Ackermann G, et al. Resistance to moxifloxacin in toxigenic Clostridium difficile isolates is associated with mutations in gyrA. Antimicrobial Agents and Chemotherapy $2001 ; \mathbf{4 5}: 2348-2353$.

6. Takeshi K, et al. Simple method for detection of Clostridium botulinum type A to F neurotoxin genes by ploymerase chain reaction. Microbiology and Immunology 1996; 40: 5-11.

7. Lindström M, et al. Multiplex PCR assay for detection and identification of Clostridium botulinum types A, B, E, and F in food and fecal material. Applied and Environmental Microbiology 2001; 67: 5694 5699.

8. Schaumann R, et al. Inducible metronidazole resistance in nim-positive and nim-negative Bacteroides fragilis group strains after several passages metronidazole containing columbia agar plates. Infection 2005; 33: 368-372.

9. Schaumann R, Laurin F, Rodloff AC. Molecular typing of clinical isolates of Stenotrophomonas maltophilia by pulsed-field gel electrophoresis and random primer PCR fingerprinting. International Journal of Hygiene and Environmental Health 2008; 211: 292298.

10. Tenover FC, et al. Interpreting chromosomal DNA restriction patterns produced by pulsed-field gel electrophoresis: criteria for bacterial strain typing. Journal of Clinical Microbiology 1995; 33: 2233-2239.

11. Tuazon CU, Miller H, Shamsuddin D. Antimicrobial activity of street heroin. Journal of Infectious Diseases 1980; 142: 944.

12. McLauchlin $\mathbf{J}$, et al. An investigation into the microflora of heroin. Journal of Medical Microbiology 2002; 51: 1001-1008.

13. Brett MM, Hallas G, Mpamugo O. Wound botulism in the UK and Ireland. Journal of Medical Microbiology 2004; 53: 555-561.

14. Merrison AF, et al. Wound botulism associated with subcutaneous drug use. British Medical Journal 2002; 325: 1020-1021. 
15. CDC. Botulism in the United States, 1899-1996. In: Handbook for Epidemiologists, Clinicians, and Laboratory Workers. Atlanta, GA: Centers for Disease Control and Prevention, 1998.

16. Critchley EM, Hayes PJ, Isaacs PE. Outbreak of botulism in north west England and Wales, June, 1989. Lancet 1989; 2: 849-853.

17. Akbulut D, et al. Wound botulism in injectors of drugs: upsurge in cases in England during 2004. Eurosurveillance 2005 ; 10: 172-174.

18. Akbulut D, Grant KA, McLauchlin J. Improvement in laboratory diagnosis of wound botulism and tetanus among injecting illicit-drug users by use of realtime PCR assays for neurotoxin gene fragments. Journal of Clinical Microbiology 2005; 43: 43424348.

19. Passaro DJ, et al. Wound botulism associated with black tar heroin among injecting drug users. Journal of the American Medical Association 1998; 279: 859863.

20. Werner SB, et al. Wound botulism in California, 1951-1998: recent epidemic in heroin injectors. Clinical Infectious Diseases 2000; 31: 1018-1024.

21. Brett MM, et al. Soft tissue infections caused by sporeforming bacteria in injecting drug users in the United Kingdom. Epidemiology and Infection 2005; 133: 575582.

22. CDC. Wound botulism among black tar heroin users - Washington, 2003. Morbidity and Mortality Weekly Report 2003; 52: 885-886.

23. Kimura AC, et al. Outbreak of necrotizing fasciitis due to Clostridium sordellii among black-tar heroin users. Clinical Infectious Diseases 2004; 38: 87-91.
24. Bundeskriminalamt. Rauschgiftjahresbericht 2002 Bundesrepublik Deutschland. (http://www.bka.de/lageber ichte/rg/2002/jahresbericht_2002.pdf). Accessed 20 March 2009.

25. European Monitoring Centre for Drugs and Drug Addiction. Annual report 2006: the state of the drugs problem in Europe (http://ar2006.emcdda.europa.eu/ en/home-en.html). Accessed 20 March 2009.

26. Schröter M, et al. Epidemiological dynamics of hepatitis $\mathrm{C}$ virus among 747 German individuals: new subtypes on the advance. Journal of Clinical Microbiology 2002; 40: 1866-1868.

27. Wylie JL, Shah L, Jolly AM. Demographic, risk behaviour and personal network variables associated with prevalent Hepatitis C, Hepatitis B, and HIV infection in injection drug users in Winnipeg, Canada. BMC Public Health 2006; 6: 229 (http://www.biomedcentral.com/ 1471-2458/6/229).

28. Gordon RJ, Lowy FD. Bacterial infections in drug users. New England Journal of Medicine 2005; 353: 1945-1954.

29. Fowler A, Mackay A. Community-acquired methicillin resistant Staphylococcus aureus pyomyositis in an intravenous drug user. Journal of Medical Microbiology 2006; 55: 123-125.

30. Gutzwiller FS, et al. Botulism: prevention, clinical diagnosis, therapy and possible threat. Deutsche Medizinische Wochenschrift 2008; 133: 840-845.

31. Cooper JG, et al. Clostridium botulinum: an increasing complication of heroin misuse. European Journal of Emergency Medicine 2005; 12: 251-252.

32. Wainberg MA. The need to promote public health in the field of illicit drug use. Canadian Medical Association Journal 2006; 175: 1395-1396. 